\title{
Mortalidade Precoce por Doenças do Coração no Brasil. Comparação com Outros Países
}

\author{
Paulo Andrade Lotufo
}

São Paulo, SP

\begin{abstract}
Objetivo - Comparar a mortalidade por doenças do coração e pela doença coronária (DC) nas faixas etárias dos 45 aos 64 anos, no período 1984-87, em capitais brasileiras com outros países.

Métodos - Foram utilizadas as estatísticas oficiais de mortalidade de 8 capitais com boa qualidade de informação: Belém, Recife, Salvador, Belo Horizonte, Rio de Janeiro, São Paulo, Curitiba e Porto Alegre e analisadas as doenças do coração [DC (410-414), mais insuficiência cardíaca (428), mais hipertensão (401-404)] e, isoladamente, a DC. A população foi a determinada nos Censos Demográficos de 1980 e 1991.

Resultados - A comparação com outros países mostrou que: a) as taxas de mortalidade por doenças do coração situam algumas cidades brasileiras na posição intermediária (Hungria, Rio de Janeiro, Finlândia, Porto Alegre e Polônia) e entre as mulheres no topo (Rio de Janeiro, Curitiba, Hungria, Porto Alegre, Inglaterra e Gales); b) DC apresentou o mesmo padrão (intermediário alto) das doenças do coração entre os homens (Finlândia, Hungria, Inglaterra e Gales, Porto Alegre e Rio de Janeiro). Entre as mulheres, repetiu-se a situação das doenças do coração (Rio de Janeiro, Curitiba, Hungria, Porto Alegre, Inglaterra e Gales).

Conclusão - A comparação com outros países das taxas de mortalidade ajustadas por idade na faixa etária dos 45-64 anos no período 1984-87 mostrou que as cidades brasileiras estudadas têm altas taxas de mortalidade para as doenças do coração, principalmente entre as mulheres, em valores tão ou mais elevados do que os da Europa e dos Estados Unidos.
\end{abstract}

Palavras-chave: epidemiologia, doenças do coração, mortalidade

\section{Premature Mortality from Heart Diseases in Brazil. An International Comparison}

Purpose - Chronic diseases have been the main cause of mortality in the Brazilian metropolitan areas since 1960. Circulatory diseases are the main causes of chronic diseases. The aim was to compare the heart disease related premature deaths in Brazil with other countries in 1984-87.

Methods - We studied the deaths from the official mortality statistics from eight metropolitan areas with good assurance of information: Belém, Recife, Salvador, Belo Horizonte, Rio de Janeiro, São Paulo, Curitiba, Porto Alegre. Death causes analyzed were coronary heart disease (410-414); and heart diseases [ischemic heart disease (410-414) plus heart failure (428) plus, hypertension (401-404)]. The population was determined from the National Brazilian Census in 1980 and 1991, in other years it was determined by linear interpolation.

Results - The international comparison showed that: a) heart disease mortality rates for Brazilian men was ranked (Hungary, Rio de Janeiro, Finland, Porto Alegre and Poland) in the medium and among women (Rio de Janeiro, Curitiba, Hungary, Porto Alegre and England and Wales) in the top; b) coronary heart diseases showed the same pattern of heart diseases for men (Finland, Hungary, England and Wales, Porto Alegre, Rio de Janeiro) and for women (Rio de Janeiro, Curitiba, Hungary, Porto Alegre, England and Wales).

Conclusions - The international comparison of ageadjusted mortality rates in the age strata 45-years-old to 64-years-old during the period 1984-87 showed that Brazilian cities had a high mortality pattern of heart diseases, similar or greater than Europe or United States, mainly among women.

Key-words: epidemiology, heart diseases, mortality

Arq Bras Cardiol, volume 70 (n 5), 321-325, 1998

Hospital das Clínicas - FMUSP

Correspondência: Paulo Andrade Lotufo - Hospital das Clínicas - Av. Dr. Enéas C. Aguiar, 155 - PAMB- AGD - S/6 - 05403-000 - São Paulo, SP

Recebido em 27/12/96

Aceito em 20/2/98
As doenças cardiovasculares constituem importante causa de mortalidade no país, representando a principal causa de mortalidade por todas as causas ${ }^{1}$. Elas assumiram um papel preponderante desde os anos $60^{2}$ nas capitais 
brasileiras e, em cidades como São Paulo, são a principal causa de morte desde o final dos anos $40^{3}$. No país como um todo, a doença cerebrovascular prepondera sobre a doença coronária (DC), exceto em cidades como São Paulo onde a DC representa contingente maior de vítimas do que a afecção cerebrovascular ${ }^{1}$.

Apesar do declínio das taxas de mortalidade por DC e cerebrovascular descritas em São Paulo ${ }^{4,5}$ e em outras capitais, a participação relativa das doenças cardiovasculares continua sendo a mais importante dentre as causas de óbito ${ }^{6}$.

A comparação internacional das taxas de mortalidade dos principais centros do Brasil com a de outros países já mostrou valores elevados de mortalidade geral ${ }^{7}$ e para todo o conjunto das doenças cardiovasculares ${ }^{8}$.

Devido às diferenças na forma de especificar o óbito por doença cardíaca na declaração, convencionou-se utilizar para comparação internacional preferencialmente à DC isolada, o conjunto que engloba a hipertensão arterial (HA), a insuficiência cardíaca(IC) e a DC, em um conjunto denominado doenças do coração ${ }^{9}$.

\section{Métodos}

As doenças do coração, conjunto composto pela DC (CID-9: 410-414); pelaIC(CID-9: 428) e pelaHA(CID-9:401404)e, isoladamente, a DC (CID-9: 410-414) foram utilizadas para comparação internacional.

Os dados de mortalidade dos países selecionados foram fornecidos especialmente pelo National Institutes of Health (Bethesda, Maryland; EUA) ${ }^{10}$, e as informações das cidades brasileiras foram obtidas dos dados de Declarações de Óbito do Sistema de Informação sobre Mortalidade (1979-90) editado em conjunto pelo Centro Nacional de Epidemiologia (CENEPI) e pelo DATA-SUS da Fundação Nacional de Saúde do Ministério da Saúde em Compact Disc Data Storage.

Devido a impossibilidade de se analisar o conjunto da federação ou uma quantidade razoável de estados, optouse pela comparação com as capitais com os melhores indicadores de qualidade de notificação, como Belém, Recife, Salvador, Belo Horizonte, Rio de Janeiro, São Paulo, Curitiba e Porto Alegre.

Limitou-se a comparação ao quadriênio 1984-1987e 'a faixa etária dos 45 aos 64 anos. Os coeficientes de mortalidade foram calculados pela divisão da somatória dos óbitos correspondente ao sexo e faixa etária nos anos de 1984, 1985, 1986 e 1987 com a somatória da respectiva população.

Devido a possibilidade de existirem taxas de mortalidade próximo em uma cidade com mortalidade geral elevadae proporção de sinais e sintomas mal definidos também elevada e outra cidade em situação oposta, todos os óbitos foram corrigidos seguindo a mesma orientação de Murray e Lopez ${ }^{11}$, ou seja, de considerar que estes apresentam a mesma distribuição da mortalidade geral, exceto pelas causas externas. Assim, para cada causa básica estudada, em cada faixa etária e sexo foram acrescentados o número de óbitos da fração atribuível a esta causa entre os sinais e sintomas mal definidos.
Para a determinação das taxas de mortalidade, utilizouse a população residente nas capitais de áreas metropolitanas obtida nos Censos Demográficos do Brasil de 1980 e 1991.

Nos anos intercensitários, as populações foram calculadas por interpolação linear simples dos dados de 1980 e 1991. A população do ano de 1979 foi calculada diminuindose dos valores correspondentes a 1980, o incremento anual do decênio 1980-89 calculado acima.

Estudaram-se as faixas etárias por sexo para comparação com outros países, utilizando-se os intervalos entre os 45 anos e os 64 anos de idade com divisões decenais (45-54 anos e 55-64 anos). Os pesos utilizados foram 0,525892 para a faixa dos 45-54 anos e de 0,474108 para a faixa 55-64 anos ${ }^{10}$.

\section{Resultados}

No sexo masculino (fig. 1), as cidades brasileiras apresentam valores elevados para o conjunto das doenças do coração, destacando-se o Rio de Janeiro, Porto Alegre e Curitiba com taxas elevadas superada pela da Hungria e próximas às da Finlândia e Polônia. São Paulo, Recife, Belém e Belo Horizonte se situam em uma posição intermediária e Salvador posiciona-se mais abaixo em valores próximos aos de Holanda e Canadá, porém distintos dos países imediatamente abaixo como Itália, Espanha e Portugal.

No sexo feminino (fig. 2), as taxas das cidades brasileiras são das mais elevadas, posicionando o país juntamente com a Hungria como os de maior mortalidade entre as mulheres e a dos Estados Unidos em posição inferior.

Se, para as doenças do coração entre os homens, as taxas de mortalidade posicionam as cidades brasileiras no topo, especificamente na DC, tal fato não ocorre com a mes-

\begin{tabular}{|c|c|c|}
\hline \multicolumn{3}{|c|}{$\begin{array}{l}\text { Quadro I - Comparação da razão de taxas de mortalidade masculina e } \\
\text { feminina para a doença isquêmica e a doença coronária (em ordem } \\
\text { crescente da razão pelas doenças do coração) }\end{array}$} \\
\hline Cidade/país & Doenças do coração & Coronária \\
\hline Salvador & 1,71 & 2,30 \\
\hline Recife & 2,06 & 2,72 \\
\hline São paulo & 2,34 & 2,84 \\
\hline Rio de janeiro & 2,39 & 2,79 \\
\hline Curitiba & 2,39 & 2,88 \\
\hline Japão & 2,46 & 3,20 \\
\hline Belém & 2,47 & 2,74 \\
\hline Belo horizonte & 2,62 & 3,36 \\
\hline Estados unidos & 2,71 & 3,41 \\
\hline Portugal & 2,83 & 3,66 \\
\hline Porto alegre & 2,95 & 3,25 \\
\hline Hungria & 2,98 & 3,39 \\
\hline Austrália & 3,23 & 3,41 \\
\hline Espanha & 3,31 & 4,77 \\
\hline Itália & 3,42 & 4,90 \\
\hline Canada & 3,44 & 3,77 \\
\hline Inglaterra & 3,50 & 3,67 \\
\hline Dinamarca & 3,64 & 3,86 \\
\hline Polônia & 3,67 & 5,85 \\
\hline Holanda & 3,80 & 4,34 \\
\hline França & 4,05 & 5,56 \\
\hline Finlândia & 5,11 & 5,57 \\
\hline
\end{tabular}




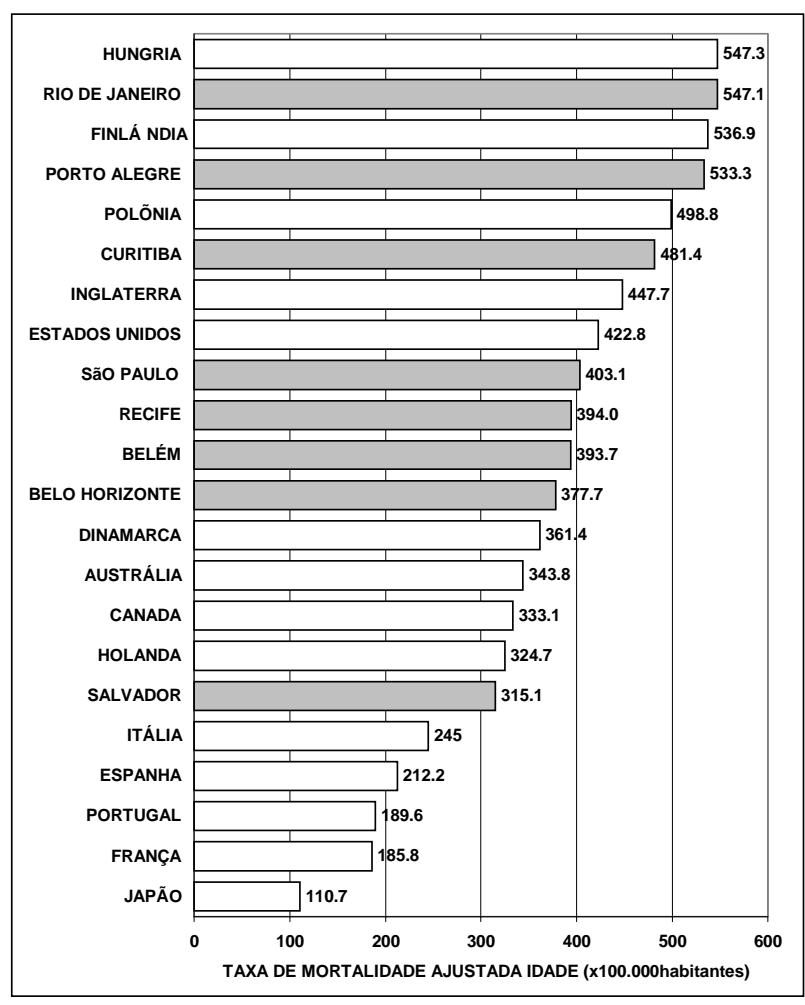

Fig. 1 - Comparação das taxas de mortalidade pelas doenças do coração na faixa etária dos 45-64 anos de idade entre cidades brasileiras e países selecionados no sexo masculino (taxa mortalidade $=\mathrm{x} 100.000$ habitantes).

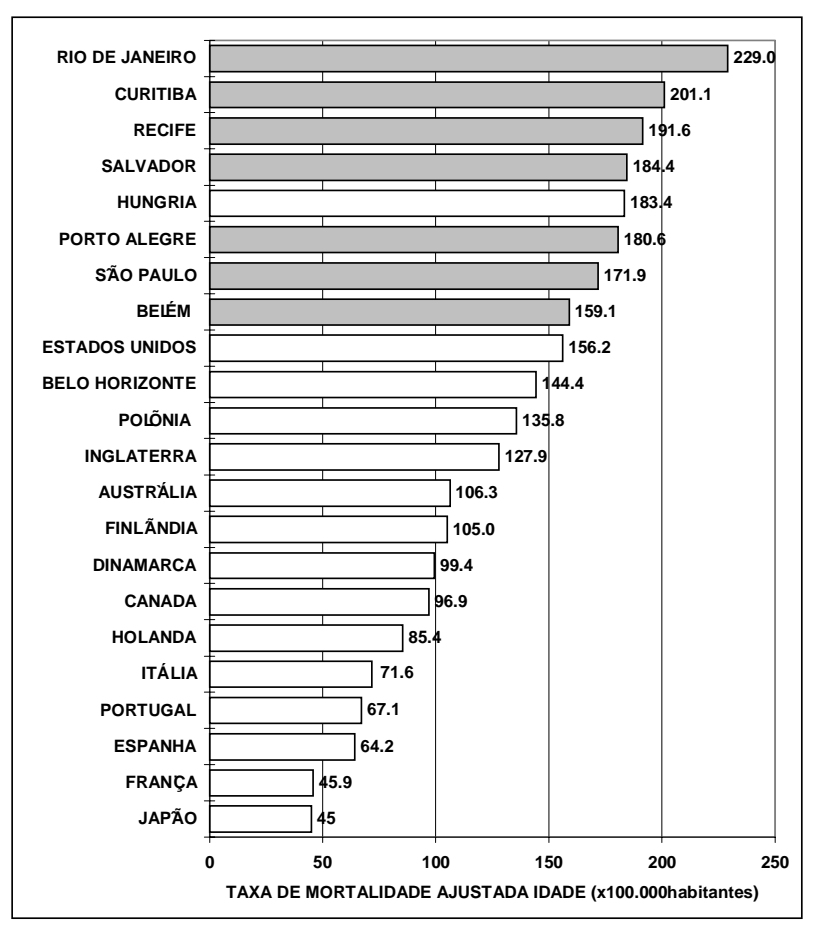

Fig. 2 - Comparação das taxas de mortalidade e pelas doenças do coração na faixa etária dos 45-64 anos de idade entre cidades brasileiras e países selecionados no sexo feminino (taxa mortalidade $=\mathrm{x} 100.000$ habitantes).

ma magnitude (fig. 3). Porto Alegre, Rio de Janeiro e Curitiba situam-se na parte intermediária alta com valores elevados; São Paulo em posição intermediária juntamente com Recife e as demais cidades (Belém, Belo Horizonte e Salvador), em posição inferior.

No entanto, para as mulheres, a mortalidade pela DC (fig. 4) é muito mais significativa, com o Rio de Janeiro e Curitiba com os valores mais elevados entre todos os locais estudados, com Porto Alegre e São Paulo com valores próximos e elevados. As demais cidades posicionam-se na parte intermediária alta do gráfico comparativo.

A observação e comparação das figuras permite concluir que o componente não-isquêmico das doenças do coração é elevado nas cidades brasileiras. Porém, a comparação dos valores isolados da DC altera pouco o posicionamento das cidades brasileiras, principalmente entre as mulheres.

No quadro I, compara-se a razão de taxas de mortalidade entre os sexos, tanto para as doenças do coração como para a coronária, mostrando que nas cidades brasileiras, a probabilidade de um homem morrer destas doenças é maior do que a de uma mulher, porém em proporção inferior à existente em outros países. Este fato ocorre, não por que a mortalidade entre os homens brasileiros é baixa, mas sim por causa das altas taxas observadas entre as mulheres das cidades brasileiras.

\section{Discussão}

Os resultados apresentados mostram que a mortalidade pelas DC no Brasil é das mais elevadas, quando comparada a de outros países e, ao contrário destes, a participação das mulheres na composição dos óbitos é das mais elevadas. Limitações de ordem metodológicas (estatísticas de saúde), epidemiológicas e demográficas devem ser ressaltadas.

As limitações das estatísticas de saúde sempre estão presentes em qualquer estudo de mortalidade. A qualidade da informação comprovada nos estudos de validação de São Paulo ${ }^{12}$ e a progressiva diminuição dos óbitos classificadas no capítulo dos Sinais e Sintomas Mal Definidos em todo país indicam melhora na qualidade e a possibilidade de comparação internacional. A comparação de países com cidades, mesmo sendo cidades de um país, possibilita viés com aumento de invasões de óbitos que são mais significativos nos estudos de neoplasias e não de doenças cardíacas, é um problema incontornável devido à impossibilidade de se analisar o Brasil como um todo.

Uma explicação demográfica das altas taxas de mortalidade pela doenças do coração nas cidades brasileiras no quadriênio 1984-87 poderia ser especulada pelo momento de análise do fenômeno epidemia da mortalidade por DC. A diversidade da tendência de qualquer (ascensão, estabilidade ou declínio) da "epidemia coronária", entre países e cidades de um mesmo país, se expressa em um instantâneo como o apresentado. Em um segundo momento (no caso, em uma década) um outro padrão poderá ser flagrado, refletindo as diversas tendências em cada sociedade ou, no caso de tendências concordantes, dos diversos graus de aceleração ou desaceleração das taxas de mortalidade.

O declínio da mortalidade pela DC foi descrita nos Estados Unidos no final da década de 60; na Europa Ocidental 


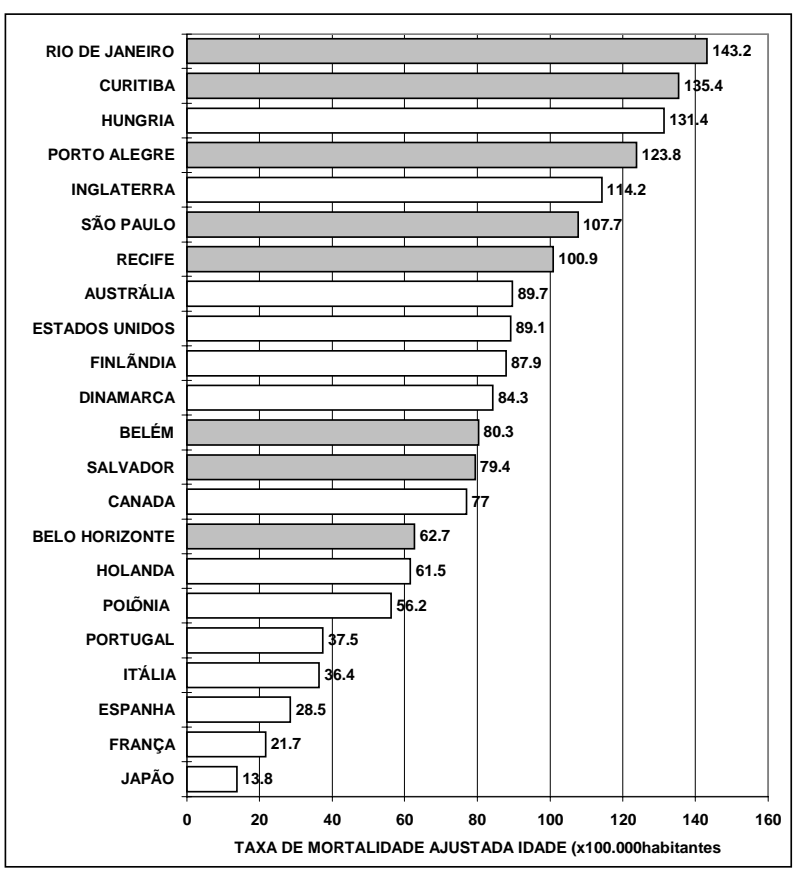

Fig. 4-Comparação das taxas de mortalidade pela doença isquêmica do coração e pela doença do coração na faixa etária dos 45-64 anos de idade entre cidades brasileiras e países selecionados no sexo masculino (taxa de mortalidade $=\mathrm{x} 100.000$ habitantes).

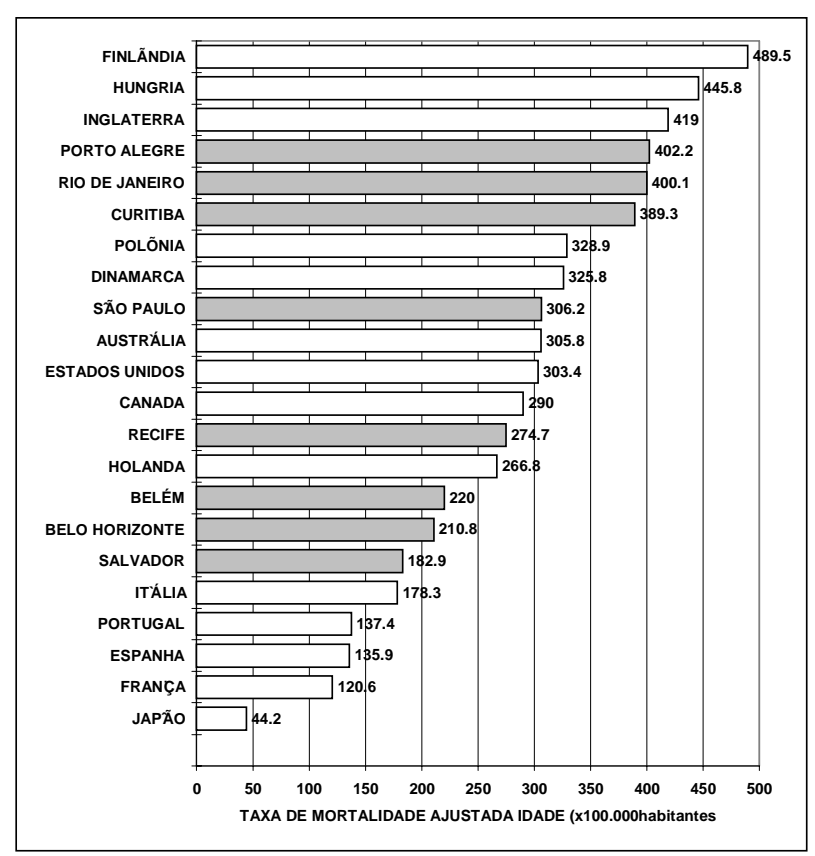

Fig. 3 - Comparação das taxas de mortalidade pela doença isquêmica do coração na faixa etária dos 45-64 anos de idade entre cidades brasileiras e países selecionados no sexo masculino (taxa de mortalidade $=\mathrm{x} 100.000$ habitantes).

em meados dos anos 70 e na Europa Oriental há aumento de taxas. No Brasil, o declínio é consistente no Estado de São Paulo somente desde o início dos anos 80 (no estudo comparativo, a capital de São Paulo encontra-se sempre com valores intermediários) ${ }^{4}$. Em outras cidades, como Porto Alegre ou Curitiba, a situação é de estabilidade, enquanto no Rio de Janeiro há aumento de taxas ${ }^{5}$.

Esta dinâmica populacional necessita ser avaliada abrangendo não somente os anos-calendário como as coortes de nascimento, método que ainda não é factível pelos poucos anos do sistema de mortalidade com cobertura nacional (início: 1977).Um óbice de caráter etiológico específico do Brasil seria o originado pela "contaminação" do conjunto doenças do coração, mais especificamente na rubrica IC pela miocardite chagásica que é classificada no capítulo das doenças infecto-parasitárias. Um estudo com delineamento ecológico, correlacionando a mortalidade por miocardiopatia chagásica (CID-9: 086) e a mortalidade por IC (CID-9: 428) com a soroprevalência para a doença de Chagas, mostrou que não existe uma associação estatisticamente significativa entre a prevalência da tripanossomíase e a mortalidade pela IC e, sim uma associação positiva e significativa da soroprevalência com a mortalidade declarada pela miocardite chagásica ${ }^{13}$, o que indicaria uma certa especificidade no diagnóstico em atestado de óbito para as miocardites.

O conjunto doenças do coração é constituído não só pela doença isquêmica do coração, como também pela HA e pela IC. A alteração de posicionamento, para cima, com a inclusão destas duas últimas doenças indica a sua importância das miocardiopatias e HA no Brasil.

A mortalidade elevada por HA não seria surpreendente, visto o observado na doença cerebrovascular. Uma questão seria o quanto que o componente IC poderia ser proveniente da hipertensão, da isquemia crônica, de miocardites virais ou da miocardite chagásica. Uma resposta mais embasada em estudos específicos de etiologia das miocardiopatias não é possível hoje, no Brasil (e, mesmo na literatura mundial), inquéritos populacionais etiológicos da IC são escassos. Em Framingham, os resultados do estudo de coorte demonstraram que a associação hipertensão-doença isquêmica seria a determinante da maioria dos casos e de que a história pregressa de angina do peito teria importância no surgimento da miocardiopatia dilatada ${ }^{14}$.

Este fato abre espaço para importante campo de investigação diagnóstica das causas de IC no país e, principalmente da influência da HA na gênese e na gravidade da história natural de outras miocardiopatias.

Por último, a questão de maior relevância reside em explicar o porquê da elevada mortalidade pela DC (e pelo conjunto das doenças do coração) no sexo feminino. Apesar do excesso de mortalidade masculina estar presente no Brasil, tal como em outros países, a chance de uma brasileira morrer de DC é maior do que a de um brasileiro da mesma idade, quando comparada com seus pares em outros países.

As especulações para tal fato podem ser muitas: maior letalidade da DC em mulheres, ausência de cultura médica em valorizar os sintomas cardíacos, aumento da prevalência de tabagismo entre as mulheres, menores cuidados na menopausa e até a questão do excesso de mortalidade masculina por causas externas (representando um fator competitivo em relação à DC). O corolário destas especulações deverá ser a realização de estudos etiológicos do tipo caso-controle com diferenciação por sexo que possam responder adequadamente a estas dúvidas. 
O fato da doença cerebrovascular apresentar quadro comparativo internacional mais destacado do que as doenças do coração, indica que talvez a chave da explicação esteja na elevada prevalência de HA no país ${ }^{15}$, complicada pelo aumento da obesidade, principalmente em mulheres ${ }^{16}$.

Apesar de limitações, como as apresentadas, o pre-

\section{Referências}

1. Estatísticas de Mortalidade, 1992 - CENEPI, Ministério da Saúde, Brasília, DF, 1996.

2. Bayer GF, Goes de Paula S - Mortalidade nas capitais brasileiras 1930-1980. RADIS-dados, 21984.

3. Mascarenhas RS, Wilson D - Diabetes Melito e Saúde Pública. Arq Hig Saúde Púb 1963.

4. Lotufo PA, Lolio CA - Coronary heart disease mortality trends in São Paulo, Brazil CVD. Epidemiol Newsletter 1994; 49: 150-1

5. Lotufo PA, Lolio CA -Tendência da mortalidade por doença cerebrovascular no Estado de São Paulo, 1970-1989. Arq Neuro-Psiquiatria 1993; 51: 441-6.

6. Lolio CA, Lotufo PA, Lira AC, Zanetta MDT, Massad E - Tendência da mortalidade por doença isquêmica do coração nas capitais de regiões metropolitanas do Brasil, 1979-89 Arq Bras Cardiol. 1995: 64: 195-9.

7. Chor D, Fonseca MJM, Andrade CR, Waissman W, Lotufo PA - Doenças cardiovasculares, panorama da mortalidade no Brasil. In: Minayo MC (org)- Os Muitos Brasis. Saúde e População na Década de 80. Hucitec/ABRASCO. São Paulo-Rio de Janeiro, 1995: 57-86.

8. Duncan B, Schimdt MI - Altos coeficientes de mortalidade em populações adultas brasileiras. RAMB 1992; 38: 138-44.

9. Epstein F - International mortality trends and secular changes. Prev Med 1983; 12: 210-7.

10. Thom TJ,Epstein F, Feldman JJ - Total mortality and mortality from heart disease, cancer, and stroke from 1950 and 1987 in 27 countries: highlights of trends and sente estudo ressalta de forma incisiva a necessidade de se incorporar as DC na agenda da saúde pública ${ }^{17}$ devendo constar nas prioridades de todas as esferas de governo e merecer a atenção de organismos não-governamentais preocupados em diminuir a desigualdade, dentro de países, entre nações. their interrelationships among causes of death. National Institutes of Health, National Heart, Lung and Blood Institute. NIH Publication 92-3088, September 1992: 92.

11. Murray, Lopes A - Patrones de distribuición mundial y regional de las causas de defunción en 1990. Bol Oficina Sanit Panam 1995: 118.

12. Laurenti R, Souza JMP, Mello-Prado MH, Gotlieb SLD - Estudo epidemiológico da morte súbita em adultos de 15 a 74 anos na cidade de São Paulo. Arq Bras Cardiol 1980; 35: 5-14.

13. Lotufo PA, Benseñor IJM-Relação entre a soroprevalência para doença de Chagas e mortalidade por miocardiopatia chagásica e por insuficiência cardíaca. Arq Bras Cardiol 1995; 65(S1): 71

14. Mc Kee PA, Castelli WP, Mc Namara PM- The natural history of congestive heart failure: the Framingham study. N Engl J Med 1971; 226: 1441-6.

15. Lessa I - Estudos brasileiros sobre epidemiologia da hipertensão arterial: análise crítica dos estudos de prevalência Informe Epidemiológico do SUS, CENEPI. Brasília, DF Mai/Jun, 1993

16. Sichieri R, Coitinho DC, Leão MM, Recine E, Everhart JE-High temporal, geographic and income variation in body mass index among adults in Brazil. Am J Public Health 1994; 84: 793-8.

17. Lotufo PA, Lolio CA - Tendências de evolução da mortalidade por doenças cardiovasculares: o caso do Estado de São Paulo. In: Monteiro CA (org) Velhos e Novos Males da Saúde no Brasil: a Evolução do País e de Suas Doenças. São Paulo: Hucitec-NUPENS/USP, 1995: 279-88. 